\title{
The Correlation Between Mean Pulmonary Arterial Pressure and NT-ProBNP in Adult Patient with Uncorrected Atrial Septal Defect
}

\author{
Muhammad Ulil Aidie Jomansyah, Dyah Wulan Anggrahini, Anggoro Budi Hartopo, Lucia Kris Dinarti \\ Department of Cardiology and Vascular Medicine, Faculty of Medicine, Universitas Gadjah Mada - \\ Dr. Sardjito Hospital, Yogyakarta, Indonesia \\ *correspondence: ulilaidie@gmail.com
}

\begin{abstract}
Background: Pulmonary Artery Hypertension $(\mathrm{PAH})$ is a group 1 of pulmonary hypertension classification. PAH can occur as a result of atrial septal defect (ASD) progression which there is chronic overvolume in the right side of the heart and pulmonary artery. PAH is debilitating vascular disease of the pulmonary circulation that leads to elevation in the pulmonary artery pressure and pulmonary vascular resistance with resultant right ventricular failure and death. PAH still have unacceptably high morbidity and mortalitydespite of various therapeutic choices. Disease severity assessment of PAH determined by symptom, exercise capacity, plasma biomarker, and haemodynamics parameter. The aim of this study is to knowing correlation of NT-proBNP with mean Pulmonary Arterial Pressure (mPAP)in adult patient with uncorrected ASD.

Method: we studied $112 \mathrm{PAH}$ in adult patients with uncorrected ASD who are also part of COHARD-PH (Congenital Heart Disease-Pulmonary Hypertension) studyat Sardjito General Hospital. PAH were diagnosed with right heart catheterization. Bloodtest for NT-proBNP, right atrium pressure and 6 minutes walk test (6MWT) were collected. Risk assessment of PAH is according to $2015 \mathrm{ESC}$ PH guideline. We performednon-parametric correlations using spearman method using SPSS version 22.

Result: one hundred and twelve patient PAH with uncorrected ASD (102 females (91\%) and 10 males $(9 \%)$, mean age $36.4 \pm 12.4$ years) underwent right heart catheterization. Patients who were in the low risk for $\mathrm{PAH}$ were $26.7 \%$, and in the moderate risk and high risk were $40.1 \%$ and $33 \%$. NT-proBNP was correlated with $\operatorname{mPAP}(r=0.639 ; \mathrm{P}<0.001)$. Correlation with mPAP was also showed by 6MWT $(r=-0.422 ; P<0.001)$ and right atrial pressure $(r=0.519 ; P<0.001)$. Conclusion: In our study, most patients were in intermediate risk for $\mathrm{PAH}$, followed by high risk and low risk. NT-proBNP, 6MWT, and right atrial pressure were correlated with MPAP in adult patient with uncorrected ASD. However, NT-proBNPis the most correlate with mPAP, followed by right atrial pressure and 6MWT.
\end{abstract}

Keywords: pulmonary artery hypertension; atrial septal defect; NT-proBNP 